\title{
Overconfidence and Entrepreneurial Choice under Ambiguity
}

\author{
Anisa Shyti ${ }^{1}$ \\ June 7, 2013
}

\begin{abstract}
Entrepreneurship studies have attributed to overconfidence decisions to start a new venture. Many decision situations, through which overconfidence is measured, entail some degrees of uncertainty (e.g., related to own skill or to competition). The aspect of uncertainty is largely neglected in overconfidence studies or entrepreneurial research. Both uncertainty and overconfidence influence individuals' likelihood perceptions. Nevertheless, these two aspects are seldom jointly investigated, and the little evidence provides inconclusive results. In this study, we experimentally investigate how uncertainty, as a property of the situation, and overconfidence, as a characteristic of decision makers' beliefs, influence choice behavior. Our findings with Executive MBA participants show that overconfident decision makers choose less uncertain options for low-likelihood outcomes and more uncertain options for high likelihood outcomes, contrary to neutralconfidence decision makers, which choices are in line with standard Prospect Theory predictions.
\end{abstract}

Keywords entrepreneurship · ambiguity attitudes $\cdot$ decision making · overconfidence

JEL classification D08 · D81 · L26

\footnotetext{
${ }^{1}$ HEC Paris, Department of Strategy and Business Policy, 1, Rue de la Liberation 78350, Jouy en Josas, France. Email: anisa.shyti@hec.edu
} 


\section{Introduction}

Entrepreneurship research attributes to overconfidence puzzling or suboptimal entrepreneurial decisions as excess entry in Koellinger et al. (2007) or perseverance in loss situations in Åstebro et al. (2007) (see also Burmeister and Schade (2007); Schade and Burmeister (2009). Entrepreneurial entry is commonly short lived half of new businesses fail within 2 years, and $67 \%$ fail or change ownership within 4 years from inception (Headd, 2003). Persistence in entrepreneurs may also not pay - $75 \%$ of entrepreneurs would be better off as wage earners and, overall, entrepreneurship reduces lifetime earnings by 35\% (Hamilton, 2000). Cooper et al. (1988) were the first to ignite a debate on entrepreneurial overconfidence, showing that a majority of entrepreneurs tend to overestimate their chances of success compared to their peers. Yet, most entrepreneurial decisions, including starting a business, are naturally exposed to uncertainty inherent the venture. Although entrepreneurship scholars recognize the importance of uncertainty in entrepreneurial decision making, aspects of uncertainty itself are mostly neglected in past studies.

Both overconfidence and ambiguity are complex phenomena. Fischhoff et al. (1977) show that people have excessive confidence in their knowledge and are ready to bet on it, accepting bets with negative expected value. Individuals can be overconfident in estimating their chances of success in competitions (Camerer and Lovallo, 1999), their performance in a given task, and their skills compared to others (Moore and Healy, 2008). Complexities of addressing individuals' overconfidence in real world matters are related to the fact that useful information to attest to overconfident decision making is available only in hindsight. There are many examples in history, on how choices that seemed overconfident and unrealistic at first, gave rise to incredible innovations and great economic returns afterwords $^{1}$. In many studies, experimental tasks used to measure overconfidence

\footnotetext{
1 "Radio has no future" - quote attributed to Lord Kelvin, Physicist and President of the
} 
require a decision under conditions of uncertainty, as betting on one's future performance. In this case, uncertainty has an endogenous source, which is the decision maker's skill. Uncertainty may also originate from exogenous sources related to a specific environment, e.g., market performance of an index, financial performance of a company, price of oil, competitors, and so on.

It could be sensible for a decision maker to act on a positive belief about future performance, but the "rationality" of this decision is available only after the consequences have materialized. From this perspective, overconfidence could be an antecedent of action, i.e., start a new venture. An omnipresent dimension of uncertainty characterizes most new venture decisions: probability distributions of outcomes are not clearly defined. Blavatskyy (2009) and Grieco and Hogarth (2009) are among the first studies that jointly consider overconfidence and ambiguity. Although both studies contribute to an interesting topic about overconfidence and ambiguity preferences in decision making, their results are inconclusive. Notwithstanding, the aspect of ambiguity seems to be under-investigated in current entrepreneurship research.

Uncertainty, the other dimension of interest in our study, is quintessentially complex. It is defined as a situation where the probability distribution of outcomes is unknown, compared to situations of risk, where probabilities are given. Business decisions seldom rely on given probabilities. The importance of uncertainty in entrepreneurial activity was understood since Knight (1921), who suggested that entrepreneurs are rewarded for bearing uncertainty rather than risk. Ellsberg (1961) showed that individuals prefer risks (known probabilities) to uncertainties (unknown probabilities), and dubbed this phenomenon ambiguity aversion, also known as Ellsberg's Paradox. Past experimental evidence shows that individuals' Royal Society, 1897. In the same year, Guglielmo Marconi, Italian inventor and entrepreneur, founded the The Wireless Telegraph \& Signal Company in Britain, and contributed in making radio a commercial success. 
attitudes towards risk are distinct from attitudes towards uncertainty ${ }^{2}$. In a recent study, Abdellaoui et al. (2011) show that ambiguity is preferred for unlikely events, and it is avoided for likely events ${ }^{3}$, thus, it emerges that under ambiguity, the pattern of likelihood distortion is similar to risk, but more exaggerated. Decades of research in decision science confirm that operationalizing and measuring uncertainty in experimental research is a complex task. Although the topic of uncertainty has raised for decades scholars' interests, we still lack consensus on appropriate methods to generate and measure uncertainty. For the purpose of our study, we use modern decision theories under uncertainty and thus contribute to the ongoing debate in the field. Furthermore, we aim to work with aspects of uncertainty embedded in a business context ${ }^{4}$ and useful to business applications.

Embracing the unknown is intrinsic in entrepreneurship, although, not all undertakings are similar in their degree of risk $^{5}$. What triggers action in conditions of uncertainty? With our study we raise a question about the antecedents of entrepreneurial decisions, the way entrepreneurs perceive environmental uncertainty, and how overconfidence contributes to shape entrepreneurial responses to uncertain business situations. More precisely, our objective is to measure ambiguity attitudes of entrepreneurs and assess the effects of overconfidence. We define overconfidence as positive beliefs about own skill, while we allow ambiguity to be an imprecision in the success rates of the venture.

Hence, we have a two-fold objective in this study: first, to measure ambiguity attitudes of decision makers; and second, to assess the impact of overconfidence

\footnotetext{
${ }^{2}$ For a comprehensive review see Camerer and Weber (1992)

${ }^{3}$ This pattern of behavior is similar to risky decision making observed in Prospect Theory (Kahneman and Tversky, 1979), which shows that decision makers are risk seeking when probabilities are low, and risk averse when probabilities are high.

${ }^{4}$ As David Schmeidler often says "The real world is not about urns and balls." Gilboa (2009)

${ }^{5} \mathrm{We}$ can illustrate this with the example of an individual, who is evaluating two alternative investment options for a fast food restaurant. He can choose between a franchise option (with relatively known rates of return), and an independent venture, with relatively unknown rates of returns.
} 
on ambiguity attitudes. We run an experiment with a unique sample of Executive MBA students in the Entrepreneurship track at HEC Paris.

Our experimental results confirm that overconfidence has an effect on ambiguity attitudes: on average, overconfident decision makers behave as ambiguity-seekers. Moreover, our findings show that overconfidence weakens the effect of ambiguity: it corrects likelihood insensitivity. Overconfident decision makers are less ambiguity seeking for low likelihood, and more ambiguity averse for high likelihood.

\section{Related Literature}

\subsection{Overconfidence}

Overconfidence, among the most studied biases in behavioral research, is a multifaceted phenomenon and bears several definitions in the literature. Overconfidence is a multi-faceted phenomenon and it can manifest in many ways. Individuals may prefer to bet on own knowledge, and too often lose (Fischhoff et al., 1977); they may overestimate their chances of success in skill competitions (Camerer and Lovallo, 1999), or overestimate their performance and ranking in tournaments (Moore and Healy, 2008). A commonly accepted definition of overconfidence is based on positive differences between estimated and actual performance. If these differences are negative, we normally refer to underconfidence. Even though pervasive, overconfidence is not a stable trait: it is shown to be related to task domain and task difficulty (Moore and Healy, 2008), familiarity, experience, expertise (Heath and Tversky, 1991), past success (Pulford, 1996).

Although overconfidence is among the most studied biases in in the psychology of judgment (De Bondt and Thaler, 1994), research is still inconclusive on its consequences for economic agents. Several theoretical studies claim that overconfidence is a positive bias (Bernardo and Welch, 2001; Compte and Postlewaite, 
2004; Heller, 2011; Van den Steen, 2011) and beneficial to the agent and the society. Yet, other studies claim that overconfidence is a bias and it needs correcting, as it may lead to wrong business decisions and be detrimental to performance (Hayward et al., 2006).

Several entrepreneurship studies associate entrepreneurial choice with overconfidence (Burmeister \& Schadde, 2007; Koellinger et al., 2007). In an experimental market entry game, Camerer \& Lovallo (1999) observe excess entry when market profits are based on future performance, as a function of skill. They report consistently lower entry levels when market profits were randomly determined. Koellinger et al. (2007) test empirically the same hypothesis on entrepreneurs. Their findings suggest that nascent entrepreneurs are more overconfident than established entrepreneurs about their own ability to run a business. They also report that overconfidence is negatively correlated with business survival.

Two main approaches have been used in experimental research to measure overconfidence: a direct judgment method (Moore \& Healy, 2008) or revealed preferences approach (Blavatskyy, 2009; Camerer and Lovallo, 1999; Grieco and Hogarth, 2009; Hoelzl and Rustichini, 2005; Fischhoff et al., 1977). In particular, in studies that employ the revealed preference approach, decision makers are offered a choice between a bet with unknown probability that depends on their performance, or a bet with a given probability. The bet that depends on decision makers performance has a main characteristics: it is ambiguous since skill in unobservable to the decision maker, and performance is unknown. Thus, this bet entails some degree of ambiguity. Individuals would plausibly take this option in case they have positive beliefs about their future performance. It seems clear that overconfidence, would lead decision makers to opt for the ambiguous bet. Most of the above mentioned studies, only address the overconfidence dimension. (Blavatskyy, 2009; Grieco and Hogarth, 2009) are among few contributions that 
jointly address overconfidence and ambiguity attitudes of decision makers. These studies will be analyzed in detail further in the paper.

\subsection{Decision Making under Risk and under Ambiguity}

In early 1921, Frank Knight suggested that entrepreneurs are rewarded for bearing uncertainty in addition to risk, but only recently researchers can analyze ambiguity in a tractable way (Abdellaoui et al., 2011). Ellsberg (1961) posits that individuals are not indifferent to ambiguity in probabilities ${ }^{6}$, as predicted in Subjective Expected Utility Theory (Savage, 1954), and they prefer options with known probabilities to options with unknown probabilities. Ellsberg's (1961) ambiguity aversion is among the most serious challenges to Expected Utility Theory. In this way, Ellsberg raised again the concerns of Keynes (1921) and Knight (1921) on the role of probabilities in decision making, and the need for a decision theory under uncertainty that extends beyond probabilistic reasoning ${ }^{7}$ (Wakker, 2010).

Violations of EUT are not limited to cases of ambiguity. Research on decision making under risk provides many examples of decisions that deviate from EUT. Allais (1953) was among the first to show that often individuals do not maximize expected utility (Wakker, 2010), although he did not challenge the role of probabilities. Analysis of decision making under risk are typically based on choices between prospects. In a typical choice problem, a decision maker faces a

\footnotetext{
${ }^{6}$ Two different streams of research developed in decision science after Ellsberg (1961): an axiomatic stream of research, aimed at modeling decision problems and an experimental stream of research, aimed at further investigating decision making under ambiguity (Curley and Yates, 1985; Einhorn and Hogarth, 1985; Fox and Tversky, 1995; Wu and Gonzalez, 1999). Choquet Expected Utility by Schmeidler (1989) and Multi-prior model Gilboa and Schmeidler (1989) are among the most prominent non-Expected Utility formal theories that model ambiguity aversion; later developments include Ghirardato et al. (2004); Klibanoff et al. (2005). Although highly sophisticated, these theories are not operational in describing and understanding individual behavior.

${ }^{7}$ Schmeidler solved this problem in 1982 , with his contribution on rank-dependent utility for uncertainty.
} 
prospect, $P$, that yields $\$ \mathrm{x}$, with probability $p$, and $\$$ y, with probability $1-p$. The utility of this prospect, $U(P)$, for the decision maker, under Expected Utility Theory (EUT), would be the sum of the expected utility of its outcomes: $U(P)=p * U(x)+(1-p) * U(y)$.

Economic applications of EUT introduce the additional assumption of risk aversion, where the shape of the utility function, $U$, is concave ${ }^{8}$.

Kahneman and Tversky (1979) introduce Prospect Theory, based on experimentally observed violations of EUT in decision making under risk. Prospect Theory shows that individuals do not perceive probabilities linearly. The common observation is that individuals overweight small probabilities and underweight large probabilities. This probabilistic distortion has been called "likelihood insensitivity" and reflects diminishing sensitivity to probabilities, or the inability to sufficiently discriminate between probability levels ${ }^{9}$. Graphically, this translates in an inverse s-shaped probability weighting function, as in Figure ??. In terms or risk behavior, under prospect theory individuals are risk seeking for small unlikely gains (i.e., state lottery), and risk averse for likely gains. Kahneman and Tversky (1979) attribute this phenomenon to cognitive psychological causes, and assume that these distortions take place during an "editing" phase, before the decision maker attaches any value to the prospects under consideration. Abdellaoui et al. (2011) show that under ambiguity individuals exhibit an exacerbated pattern of likelihood distortion compared to risk. This translates in a higher likelihood insensitivity when probabilistic information is ambiguous ${ }^{10}$. Their weighting function is flatter, as a result of the higher curvature, which is the manifestation of the probability insensitivity.

\footnotetext{
${ }^{8} u^{\prime \prime}<0$, or negative second derivative.

${ }^{9}$ According to Peter Wakker (2010) likelihood insensitivity is irrational and can be corrected through incentives and learning.

${ }^{10}$ David Budescu suggests the use of the term "vagueness", as more approriate, but most of the literature refers to "ambiguity".
} 
Of the most striking findings of this literature is that attitudes towards risk and ambiguity are not stable traits, but domain-specific, sensitive to likelihood levels and to sources of uncertaintyThis goes partially against most theories that assume that decision makers are risk averse or ambiguity averse and that these attitudes are invariant. We reveal here that one of our objectives is to understand whether overconfidence may influence one of these aspects.

\subsection{A Psychometric Perspective on Ambiguity}

Ambiguity aversion is not new in psychology research. During the late 40ies, researchers in the field developed self-reported psychometric scales to measure intolerance to ambiguity (hereafter, IA). Major contributions rank works by FrenkelBrunswik (1949), the first one to coin the IA concept and scale. Budner (1962) refined the scale and interpretation of IA, and Mac Donald Jr (1970) made additional changes to the psychometric scale. In Budner (1962) view, IA refers to the tendency of individuals to interpret an ambiguous situation as a source of threat (Budner, 1962; Mac Donald Jr, 1970) ${ }^{11}$, with reactions manifesting through cognitive, emotional and behavioral aspects ${ }^{12}$.

Early entrepreneurship applications of IA include Schere (1982), who documented that entrepreneurs score high in ambiguity tolerance, and Dollinger (1983), which results did not confirm Schere's findings. The topic was not raised again in entrepreneurship studies, until recent applications of Dollinger et al. (1995); Tajeddini and Mueller (2009); Teoh and Foo (1997). Yet again, this research does not lead to robust conclusions on how entrepreneurs perceive and act under ambiguity,

\footnotetext{
${ }^{11}$ Budner (1962) defines an "ambiguous situation as one which cannot be adequately structured or categorized by the individual because of the lack of sufficient cues or situations characterized by novelty, complexity, or insolubility." (p. 30).

${ }^{12}$ Fifty years later, Freeston et al. (1994) developed intolerance to uncertainty (IU), a different, yet related psychometric scale comapred to IA (Grenier et al., 2005; Carleton et al., 2010). The main difference between IA and IU is attributed to their time-orientation (Grenier et al., 2005)
} 
and how their decisions differ compared to managers McLAIN (2009).

\section{Theory}

This study points to the fact that entrepreneurs start a new venture with only a vague knowledge of their likelihood of success. Most entrepreneurs lack experience, face conflicting or insufficient (statistical) evidence, which makes it difficult to define precise prediction on success. Difficulties related to estimates of likelihood success for a new venture are also related to the aggregation that the notion of success incorporates. Yet, most entrepreneurs feel that their fund raising or marketing campaigns, the VCs decisions concerning their start-up, will be successful and will mostly depend on their skills. Thus, the important role of overconfidence and decision making under uncertainty.

Past studies that have measured overconfidence through a revealed preferences approach, have mostly used the following procedure. Decision makers are presented with a skill task. Afterwards, they are asked to bet on a prospect with given probability, or on their performance. In psychology studies, if the person bets on his performance (or unknown-probability) he is classified as overconfident (and underconfident if he chooses the known probability option), but he is also seeking ambiguity $^{13}$. This pattern of choice represents a reversal of Ellsberg's Paradox. Thus, this type of choice problem, and results thereby, contain a compound effect of overconfidence and ambiguity preferences. We are unable to distinguish whether the decision is due to overconfidence or ambiguity preferences, and to what extent.

Blavatskyy (2009) and Grieco and Hogarth (2009) are among few studies that jointly investigate overconfidence and preferences for ambiguity. In Grieco and

\footnotetext{
${ }^{13}$ Heath and Tversky (1991) suggest that people tend to become ambiguity seeking when the source of uncertainty is their own knowledge (see also Kilka and Weber (2001)), and they formulate the "competence hypothesis". Yet, their method relies on the revealed preference approach, where individuals need to bet on their performance in a test.
} 
Hogarth (2009) overconfidence and ambiguity attitudes are confounded: participants have to bet on their own performance, without knowing how well they did. Their results point to a small but significant effect. Blavatskyy (2009) measures overconfidence and ambiguity attitudes independently, but he finds no association between ambiguity preferences and overconfidence. Both studies give insightful information on available methodologies, but their results are inconclusive.

We may argue that the decision makers' prior beliefs may account for their revealed preferences. In the case of overconfidence, the decision maker has a prior belief regarding his skill, and he has experience on how this skill has served him in the past. Skill (broadly defined, including ability, knowledge, etc.) could be conceptualized as an endogenous source of uncertainty ${ }^{14}$. Whereas, a prospect with risky, ambiguous or unknown probability, which is not related to skill, could be referred to as an exogenous source.

Overconfidence has been related to knowledge or familiarity with a task (Heath and Tversky, 1991), to levels of expertise (Griffin and Tversky, 1992), to feedback or past success (Pulford, 1996; Hilary and Menzly, 2006). Feedback was also successfully used in Krueger and Dickson (1994) to enhance self efficacy and evaluate its effect on risky decisions. We believe that feedback is a powerful tool, which can greatly influence motivation and self-perception, and subsequent decisions under conditions of uncertainty.

Performance feedback on a skill-related task, may also influence confidence. Positive feedback, as positive reinforcement may produce overconfidence, whereas negative feedback may produce underconfidence (Pulford, 1996). Hence, it is plausible that an easy task will produce the psychological state of overconfidence, whereas a difficult task would produce a state of underconfidence. This hard-easy effect was confirmed in several studies about absolute overconfidence (Gigerenzer

\footnotetext{
${ }^{14}$ The introduction of the term source of uncertainty is originally attributed to Amos Tversky in the 1990s, and it refers to a mechanism that generates uncertainty.
} 
et al., 1991; Stankov, 2000), and on relative overconfidence ${ }^{15}$ (Hoelzl and Rustichini, 2005). Thus, overconfidence (underconfidence) is for us the psychological state of the decision maker, following positive (negative) performance feedback based on skill.

If both overconfidence and ambiguity contribute to a distortion in overall likelihood perception, and thus determine choice behavior, we would like to disentangle the effect of overconfidence from the effects of ambiguity. In particular, we would like to establish a causal link between overconfidence and ambiguity attitudes of decision makers.

In our study we consider overconfidence as a property of decision makers' prior beliefs. We expect this property, as an antecedent of choice, to impact behavior under ambiguity. Our theory posits that the effects of overconfidence may manifest in the shape of the weighting function of Prospect Theory (Kahneman and Tversky, 1979).

\subsection{A Measure for Ambiguity Attitudes}

Budescu et al. (2002) develop a measure for ambiguity attitudes of decision makers, based on a generalized version of Prospect Theory (Kahneman and Tversky, 1979), with the addition of a well-defined parameter to accommodate decision makers' attitudes towards ambiguous probabilities. In this study, decision makers are asked to evaluate through certainty equivalents $(C E),{ }^{16}$ a prospect, $P$, that yields $\$ \mathrm{x}$, with probability $p$, and $\$ \mathrm{y}$, otherwise. Under Prospect Theory, the value of this prospect depends on decision maker's utility function, $U(x)$, and probability weighting function, $f(p)$. The value of the prospect for the decision maker would be the same as for the $C E$, as follows:

\footnotetext{
${ }^{15}$ The literature presents quite conflicting, yet neglected, evidence on the hard-easy effect.

${ }^{16} \mathrm{~A}$ certainty equivalent is a sure amount of money equally desirable for the decision maker, compared to a lottery or a risky prospect
} 


$$
U(P)=f(p) * U(x)+[1-f(p)] * U(y)=U(C E)
$$

In Budescu et al. (2002) study, it is assumed that the same parameters and functions that describe decision makers responses to precise prospects, also describe their responses to the ambiguous prospects. Thus, ambiguity is operationalized through probability intervals, $[\underline{p}, \bar{p}]$, where $\underline{p}$ is the lower bound of the interval, and $\bar{p}$ is the upper bound of the interval ${ }^{17}$.

The ambiguity parameter captures how an individual summarizes the given probability interval, $[\underline{p}, \bar{p}]$, through a weighted average of its endpoints. This parameter operates within the argument of the probability weighting function of Prospect Theory.

The evaluation of a normalized prospect $(y=0)$, Budescu et al. (2002) specification is:

$$
U(x) * f\left[w_{p} \underline{p}+\left(1-w_{p}\right) \bar{p}\right]=U(C E)
$$

$C E$ is the certainty equivalent of prospect $P$

$U(C E)$ and $U(x)$ are utilities of the certainty equivalent and outcome $x$

$[\underline{p}, \bar{p}]$ is the probability interval of prospect $P$

$f($.$) is the probability weighting function$

$w_{p}$ is the ambiguity parameter

This model distinguishes between the effects of probability, captured by $f(p)$, the utility of monetary outcomes, captured by $U(x)$, and ambiguity attitudes per se, captured by the parameter $w_{p}$.

Our goal is an in-depth, precise measurement of individuals' ambiguity atti-

\footnotetext{
${ }^{17}$ For a similar approach, see Curley \& Yates, 1985; Gonzales-Vallejo, Bonazzi, \& Shapiro, 1996; Kuhn \& Budescu, 1996
} 
tudes. Also, we aim to adopt a revealed choice approach, whereas a task that requires the expression of certainty equivalents requires a commitment towards judgment ${ }^{18}$.

We adapt Budescu's et al. (2002) model, in order to introduce binary choices between an ambiguous prospect $(\$ \mathrm{x},[\underline{p}, \bar{p}])$, and a precise prospect $\left(\$ \mathrm{x}, p_{r}\right)$, where $p_{r}$ is the probability of a risky choice. We introduce a partition $n$ to the probability interval ${ }^{19}$. We allow the probability of the risky prospect to vary within the endpoints of the probability interval, thus creating a battery of $n$ risky prospects. The indifference point, $p_{r}^{*}$, allows us to elicit the ambiguity parameter $w_{p}$. In our version, indifference means that the ambiguous and precise prospects are equally valuable to the decision maker, thus they have equal utilities.

At the indifference point, $p_{r}^{*}$, the utility of each prospect is equal to the utility of each outcome multiplied by its weighted probability:

$$
U(x) * f\left[w_{p} \underline{p}+\left(1-w_{p}\right) \bar{p}\right]=U(x) * f\left(p_{r}^{*}\right)
$$

We can simplify $U(x)$, since it operates on both sides of the equation:

$$
f\left[w_{p} \underline{p}+\left(1-w_{p}\right) \bar{p}\right]=f\left(p_{r}^{*}\right)
$$

Also, since $f(p)$ is the same on both sides, we can apply the inverse $f^{-1}$, on both sides:

$$
w_{p} \underline{p}+\left(1-w_{p}\right) \bar{p}=p_{r}^{*}
$$

This approach is mathematically feasible, given Budescu's (2002) assumptions:

\footnotetext{
${ }^{18}$ Although a task involving certainty equivalents is cognitively less demanding for participants.

${ }^{19}$ The partition of the probability interval is arbitrary, and depends on precision that we want to achieve with our measurement.
} 
the same utility and probability weighting functions operates for precise and ambiguous probabilities. The explanation is natural for the utility function, since the outcome is identical and precisely defined for the ambiguous and the risky prospect. Regarding the probability weighting function, a similar logic applies: the probability weighting function that applies to point probabilities, will as well apply to the focal points of a probability interval. As such, being the focal points of the probability intervals known, the decision maker will transform these points through the same function he would transform precise probabilities. Our own specification maintains all the original properties of the Budescu et al. (2002) model.

\subsection{The Ambiguity Parameter}

With the above procedure we can efficiently elicit $w_{p}$, which provides a precise measure of decision maker's attitude towards probabilistic ambiguity.

Ambiguity attitudes are classified according to the values of the ambiguity parameter $w_{p}$. If an individual does place a higher weight to the lower bound of the probability interval, he is classified as ambiguity averse, and $w_{p}$ is higher than .5. Similarly, if an individual does place a higher weight to the upper bound of the probability interval, he is classified as ambiguity seeking, and $w_{p}$ is below .5. If the individual summarizes the probability interval with its midpoint, by placing equal weights to the lower and upper bound of the probability interval, he is classified as neutral to ambiguity, and $w_{p}$ in this case is exactly .5.

Worth of note is the fact that ambiguity attitudes elicited through this model could be a function of optimism or pessimism of decision makers. More on this point will be addressed in the discussion section of the paper. 


\subsection{Hypothesis Development}

Given the competence hypothesis of Heath and Tversky (1991), "when the source of uncertainty is the own skill, people tend to become more ambiguity seeking", we expect overconfident decision makers to be more ambiguity seeking. Still, we believe that overconfidence is a broader construct than the competence hypothesis (as shown in Ellsberg, 1961). This is partly confirmed by the fact that both Heath and Tversky (1991) and Griffin and Tversky (1992) find that individuals in the high-knowledge condition, the more competent ones, are also more overconfident. Thus, the feeling of being competent did not make overconfidence disappear.

\section{Hypothesis 1 - Overconfidence and ambiguity}

Overconfidence, on average, will lead to ambiguity seeking behavior.

Findings in Abdellaoui et al. (2011) show a rich pattern of ambiguity attitudes: individuals are ambiguity seekers for unlikely gains, and ambiguity averse for likely gains (Wakker, 2004; Wu and Gonzalez, 1999). We expect the psychological state of overconfidence to to have an impact on ambiguity attitudes. In particular, we expect overconfidence to weaken, the effect of ambiguity in decision making.

\section{Hypothesis 2 - Overconfidence and likelihood level}

2A: Overconfident decision makers will exhibit less preference for ambiguous prospects than precise prospects for low likelihood levels, and more preference for ambiguous prospects than precise prospects for high likelihood levels.

2B: This correction will not occur for neutral-confidence decision makers.

In some studies, overconfidence is measured through decision makers' confidence intervals about their knowledge. It emerges that overconfident decision 
makers hold narrow beliefs ${ }^{20}$. We aim to detect an effect of "endogenous narrow beliefs" on degrees of exogenous ambiguity.

\section{Hypothesis 3 - Overconfidence and degree of ambiguity}

Overconfident decision makers will exhibit greater preference for ambiguous prospects when facing a higher degree of ambiguity (wider probability interval).

We introduce a working hypothesis on ambiguity attitudes and stakes. We only know decision makers' reactions to stakes in studies assessing risk attitudes. It seems reasonable to speculate that decision makers will exhibit more ambiguity aversion when stakes are higher (if uncertainty is a generalized case of risk). We build this hypothesis accordingly.

\section{Hypothesis 4 - Overconfidence and stakes}

We expect decision makers to be more ambiguity averse for high stakes compared to low stakes.

\section{Experimental Design}

In order to establish a causality link between overconfidence and ambiguity, we prime our participants with three between-subjects confidence treatments (similar approach in Bolger et al. (2008)). Our three priming treatments consisted in manipulating task difficulty and providing to participants feedback accordingly. Overconfidence priming consisted in high-performance feedback on an easy test, neutral priming consisted in average-performance feedback on a medium test, and

\footnotetext{
${ }^{20}$ They overestimate the precision of their knowledge, what Moore and Healy (2008) call overprecision.
} 
underconfidence priming consisted in low-performance feedback on a difficult test. Test feedback was aimed at boosting the effect of the treatment, and not simply rely on participants performance estimates. The test consisted in ten selected questions, for each difficulty level, from the "Tons o' Trivia" quiz of Moore and Healy (2008).

After this part of the experiment participants were called to express their preferences on pair-wise prospects between an ambiguous prospect $(\$ \mathrm{x},[\underline{p}, \bar{p}]$, which obtains $\$ \mathrm{x}$ with an unknown probability within the given interval, 0 otherwise) and a risky prospect $\left(\$ \mathrm{x}, p_{r}\right)$. Choices in this part of the experiment were framed as investment decisions.

This is a $3 \times(2 \times 2 \times 3)$ mixed design, where only confidence priming is a betweensubjects factor. The choice task has a counterbalanced design and investment decisions were presented in eight different orders. For an example of choice task refer to Figure 1.

Figure 1: Illustration of prospects

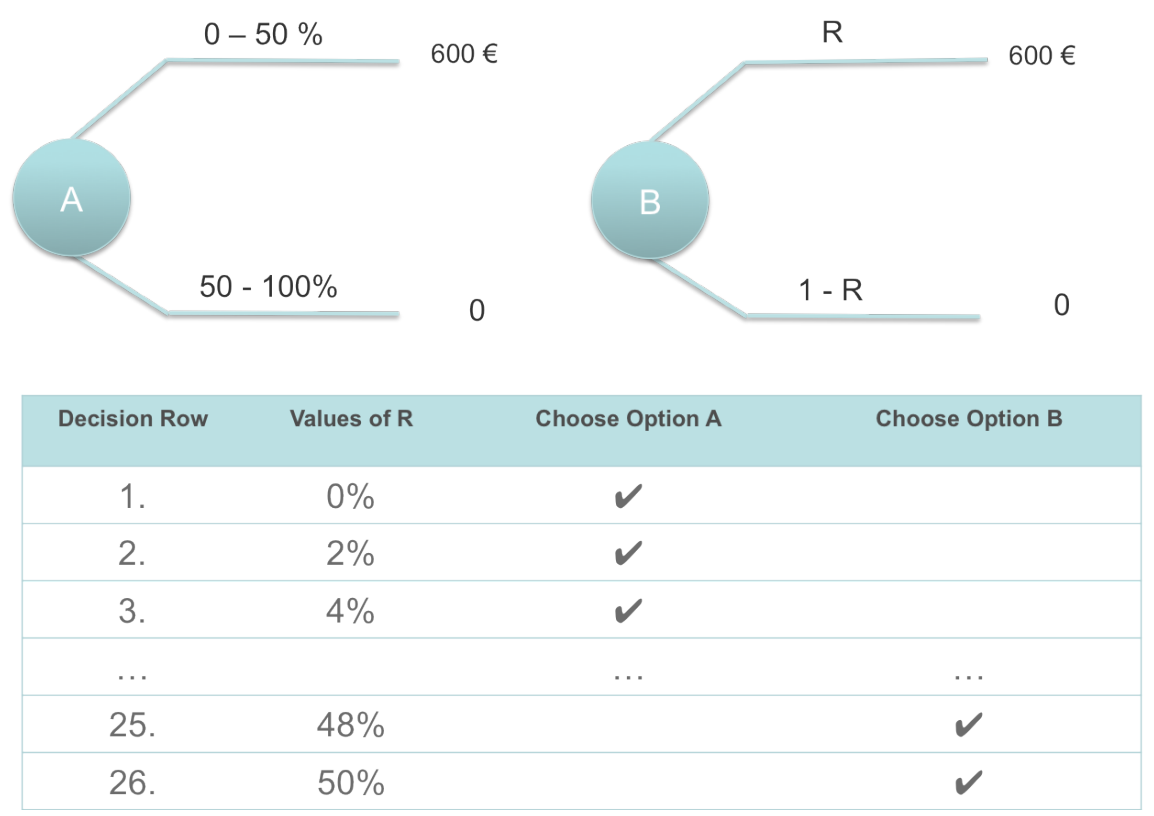


Likelihood levels. Another experiment stimulus is the likelihood level of outcomes. In order to manipulate the likeliness of each outcome, we center probability intervals in three different midpoints or likelihood levels, respectively .25 for low likelihood, .5 for moderate, and .75 for high likelihood.

Degree of ambiguity. We manipulate the degree of ambiguity by varying the width of the probability interval. Narrow intervals (of length .2) represent less ambiguous situations, whereas wide intervals (of length .5) represent more ambiguous situations. The partition $n$ is equal to .02 in both cases. This allows a refined identification of decision makers' indifference point between the ambiguous and the risky prospect. For each investment decision, participants compare the ambiguous prospect with a series of risky prospect, where the extreme values of the risky probability $p_{r i}$ are the natural boundaries of the probability interval of the ambiguous prospect. We assume uniform probability distribution inside each probability interval.

Stakes. Outcomes are defined in the gains domain only. We introduced a high stakes condition and a low stakes condition. Prospects across stakes conditions had constant expected value, computed at the midpoint of the probability interval.

The expressed indifference, or the switching point from the ambiguous prospect to the precise prospect, is our variable of interest. Through participants' choices, we are able to estimate the ambiguity parameter as shown previously. Participants were presented with 12 investment decisions, with longer and shorter probability intervals, respectively with 11 or 26 binary choices. Each line represented a jump of $2 \%$ in the probability of the risky option. Each participant was asked to express one indifference for each investment decision, i.e., start with the ambiguous 
prospect then crossover to the risky one. Thus, we have twelve indifferences for each participant.

From a stochastic dominance perspective, for a given probability interval, even an extremely ambiguity-averse decision maker should choose the ambiguous prospect in the first-row choice ${ }^{21}$, where the probability of the risky prospect, $p_{r}$, coincides with the lower bound of the probability interval, $\underline{p}$. In this way, the ambiguous option yields at least probability $\underline{p}$ or a higher probability. Likewise, even the most ambiguity-seeking decision maker should choose the risky prospect in the last-row choice option, where the probability of the risky prospect, $p_{r}$ coincides with the upper bound of the probability interval, $\underline{p}$, in which case, the risky prospect yields the highest precise probability. A similar logic applies to the entire battery of risky prospects compared to the ambiguous prospect, also away from the boundaries. Respecting this logic ensures a unique switching point. Moreover, choices that are inconsistent with the described pattern represent a violation of stochastic dominance.

\subsection{Participants, Procedure, and Instructions}

\subsubsection{Participants}

We rely on a unique sample of participants. The experiment was run with 40 Executive Education participants ${ }^{22}$ that selected the Entrepreneurship and Innovation track. The Executive Education participants were informed and invited to participate in our study in the morning of their Entrepreneurship \& Innovation seminar. Participation was voluntary. They were told that two of them would be selected at random to play for payoff one of the prospects in their booklets. Payoff was a function of their choices and of chance. The selected participant could

\footnotetext{
${ }^{21}$ Given a set of binary choices, or investment decision

${ }^{22}$ In our institution, during May 2011
} 
earn up to 600 Euros. Participants were between 32 and 49 years old, with an average age 39. Demographics of our sample show that $95 \%$ of the participants have more than 10 years work experience; 15\% are currently self-employed and $43 \%$ declare to have been business owner before. The yearly individual revenue of our participants is quite interesting: $21 \%$ falls in the 45K-90K Euros and another $20 \%$ declares individual yearly revenues above $160 \mathrm{~K}$ Euros. $95 \%$ of the sample has more than 10 years of work experience, and $62 \%$ holds a master's degree. Women were underrepresented in this group (12\%).

\subsubsection{Procedure}

The experiment was run in a plenary session with paper and pencil. Student that expressed the desire to participate in the study, filled the "Tons o' Trivia" quiz (selected questions from Moore \& Healy, 2008) at the end of the morning session. They were told that the second part of the experiment would take place at the end of the afternoon class session. Executives that decided to participate to the second part were distributed the feedback of their morning quiz and the booklet with the choice tasks. Instructions were read aloud by the experimenter and a hard copy was distributed with each booklet. On average, the first part of the experiment took 10 minutes to complete and the second part required 50 minutes.

In our experimental session, participants began by indicating their preference between option A or option B for each ordered choice list of the twelve investment decisions, with the understanding that one of these choices would be selected at random ex-post. If the participant had selected option $B$, the probability would be given and the prospect would be played for payoff. If the participant had selected option A, the probability would be randomly determined within the given probability interval, then the prospect would be played for payoff. The assumption underlying this point, also for practical matters, was uniform probability distribu- 
tion inside each probability interval. This procedure satisfies the BDM-incentive compatibility elicitation method (see Becker, DeGroot, \& Marschak, 1964).

Participants had a hard copy of the instructions, provided with their experimental booklet.

One possible limitation of this approach could be that incentives are likely to be diluted first, by the random selection of one investment decision and then by the random selection of just two participants for payoff (Starmer \& Sugden, 1991). Although, participants were engaged through the experiment and showed great interest during the debriefing session.

\section{Results}

Before proceeding with more aggregated analysis, we first summarize the raw data as in the Figures 2 and 3. These graphs represent the proportions (\%) of ambiguous choices for each experimental condition (for a similar approach, see $\mathrm{Wu}$ and Gonzalez (1996)). This very simple representation shows the different patterns of ambiguous choices through confidence priming treatments and other experimental conditions. The well-distinguished red curve in these graphs, represents the proportion of ambiguous choices of overconfident decision makers. Overconfident decision makers choose less ambiguous options for low likelihood prospects (probability interval midpoint .25), compared to the other two groups; whereas, for the moderate and high likelihood prospects (respectively probability interval midpoints .5 and .75) overconfident decision makers increase the proportion of chosen ambiguous prospects. Similar to the approach of Wu and Gonzalez (1996) for risk, the proportion of ambiguous choices would drive the curvature of the weighting function. 
Figure 2: Ambiguous choices (\%) for narrow probability intervals

Narrow intervals, low EV

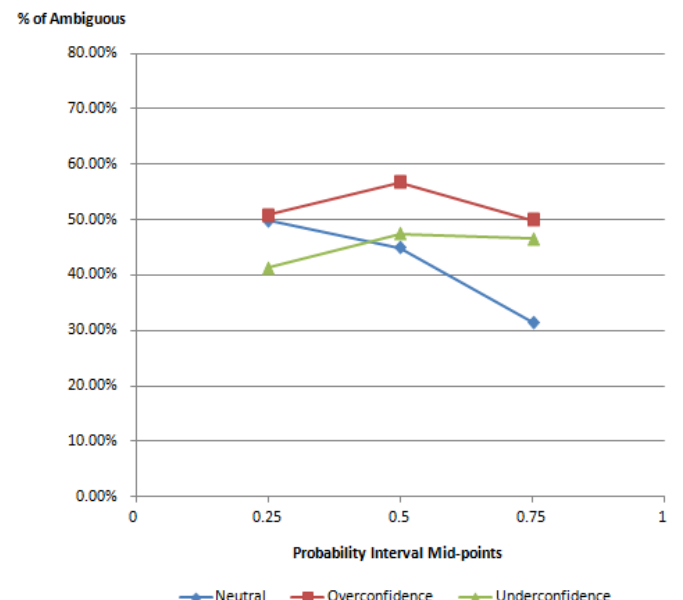

Figure 3: Ambiguous choices (\%) for wide probability intervals

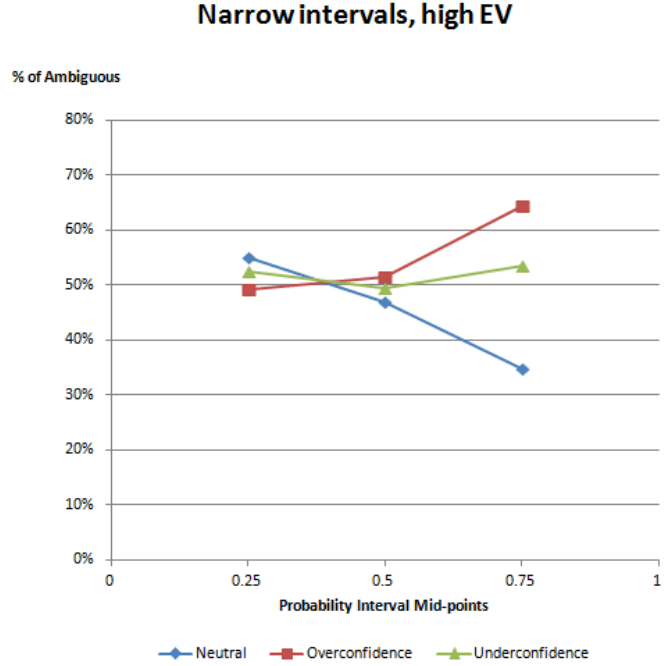

$\rightarrow$ Neutral $\rightarrow$-Overconfidence $\rightarrow-$ Underconfidence

Narrow intervals, high EV
Wide intervals, low EV

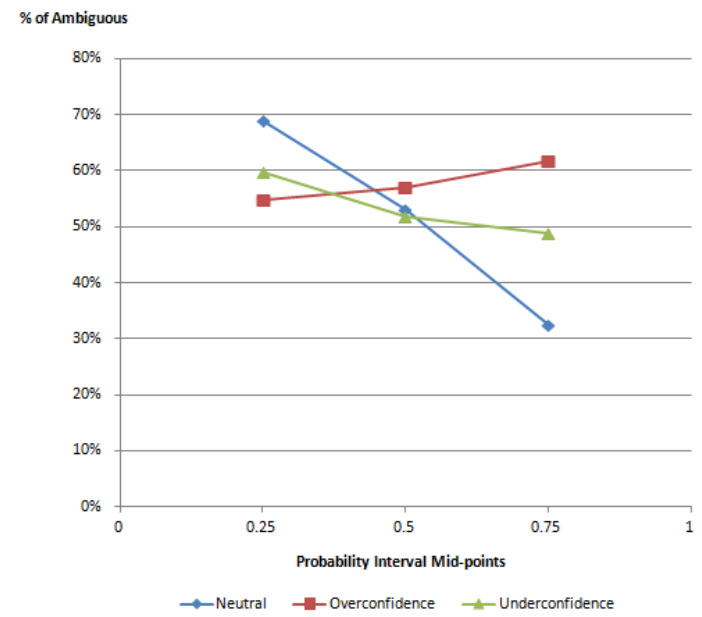

Wide intervals, high EV

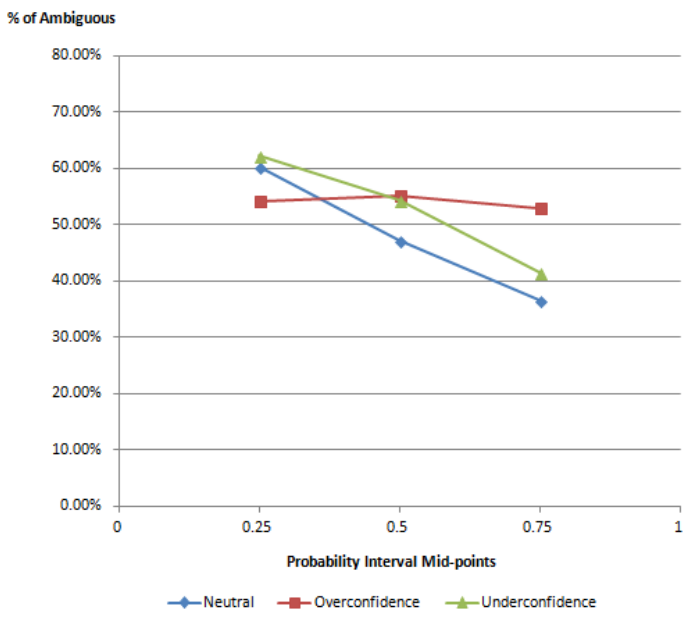

The results of our One-way ANOVA confirm our Hypothesis 1: when primed with overconfidence participants tend to prefer ambiguity rather than risk. Given our measure of ambiguity attitudes, our overconfident (OC) decision behave as 
ambiguity seekers, with an ambiguity parameter $w_{p}=.46$, (significantly different from $0, \operatorname{Pr}(\mathrm{T}>\mathrm{t})=.000)$, significantly lower than $.5(\operatorname{Pr}(\mathrm{T}<\mathrm{t})=0.0007)$. Our results show that our neutral-confidence (NC) decision makers are ambiguity averse, with $w_{p}=.534$ (significantly different from $0, \operatorname{Pr}(\mathrm{T}>\mathrm{t})=.000$ ), significantly higher than $.5(\operatorname{Pr}(\mathrm{T}>\mathrm{t})=0.0017)$. Participants primed with underconfidence (UC) exhibit an ambiguity parameter $w_{p}=.49$ (different from 0 at $5 \%$ significance), non-significantly different from $.5(\operatorname{Pr}(\mathrm{T}>\mathrm{t})=0.9270)$. Thus, our UC participants are ambiguity neutral.

Likelihood levels (H2). Our Hypothesis 2 is also supported: as expected, our analysis show that decision makers exhibit ambiguity seeking for small likelihood levels and ambiguity aversion for high likelihood levels (H2a). Overconfidence appears to weaken the effect of ambiguity $(\mathrm{H} 2 \mathrm{~b})$, at least for moderate and high likelihood levels. OC decision makers are consistently ambiguity seekers, compared to $\mathrm{NC}$ and UC groups. In particular, NC decision makers switch from ambiguity neural behavior for low likelihood levels to ambiguity averse for high likelihood level $(\operatorname{Pr}(\mathrm{T}<\mathrm{t})=0.0822)$. Likewise, UC decision makers switch from ambiguity seeking for low likelihood levels to ambiguity averse for high likelihood levels $(\operatorname{Pr}(\mathrm{T}<\mathrm{t})=0.0485)$. The $\mathrm{OC}$ decision makers seem to be more consistent in their ambiguity attitudes throughout likelihood levels.

Degree of ambiguity (H3). The degree of ambiguity does not seem to affect ambiguity attitudes of our participants (H3). However, participants in the neutral group (NC) exhibit less ambiguity aversion when they face wide intervals compared to narrow intervals $(\operatorname{Pr}(\mathrm{T}>\mathrm{t})=0.040)$. OC decision makers are consistently ambiguity seeking, disregarding the degree of ambiguity. UC decision makers are ambiguity seekers for narrow intervals and they behave as ambiguity neural for 
wide intervals, although effects are non-statistically significant. These results show that overconfidence contributes to consistency of behavior disregarding ambiguity. For similar degrees of ambiguity, OC decision makers exhibit ambiguity seeking behavior compared to the other groups (NC, UC).

Stakes (H4). Stakes seem to have the expected effect on ambiguity attitudes. Pooled data show that for low stakes decision makers are ambiguity neutral: the ambiguity parameter is not significantly different from $.5(\operatorname{Pr}(\mathrm{T}>\mathrm{t})=0.2354)$. For high stakes decision makers become slightly ambiguity averse $(\operatorname{Pr}(\mathrm{T}>\mathrm{t})=$ 0.0532). Though, OC decision makers are consistently ambiguity seekers, for both low and high stakes compared to NC decision makers, which are consistently ambiguity averse $(\operatorname{Pr}(\mathrm{T}<\mathrm{t})=0.0056)$. Interestingly, the UC decision makers switch from ambiguity seeking for low stakes to ambiguity averse for high stakes $(\operatorname{Pr}(\mathrm{T}$ $<\mathrm{t})=0.0167)$.

\subsection{Overonfidence and Weighting Functions}

In order to test the effect of overconfidence on ambiguity attitudes compared to risk attitudes, we assume a linear weighting function, also called neo-additive family, as in Abdellaoui et al. (2011), for both risk and ambiguity. In this part of the analysis, we relax the assumption of Budescu et al. (2002)model that the parameters of weighting functions for risk and ambiguity are the same. Linear weighting functions are a simple, but extremely useful family of probability weighting functions (6):

$$
f(p)=b+a p
$$


$f(p)$ is the weighting function

$b$ is the optimism index, which accounts for elevation

$a$ is the insensitivity index, the slope of the curve

At the indifference point, we have equal utilities of the prospects:

$$
\begin{gathered}
U(x) * f\left(p_{a}\right)=U(x) * f\left(p_{r i}\right) \\
b_{a}+a_{a} p_{a}=b_{r}+a_{r} p_{r}
\end{gathered}
$$

Where $b_{a}$ is the optimism index for ambiguity, and $b_{r}$ is the optimism index for risk; $a_{a}$ is the insensitivity index for ambiguity, and $a_{r}$ is the insensitivity index for ambiguity; $p_{a}$ is the probability of the ambiguous option and $p_{r}$ is the probability of the risky option

We can rearrange the above equation, in order to estimate the ratio of the weighting function parameters:

$$
p_{r i}=\left(\frac{a_{a}}{a_{r}}\right) p_{m i d}+\frac{b_{a}-b_{r}}{a_{r}}
$$

Where, $p_{\text {mid }}$ is the midpoint of the given probability interval. This way, we can compare the switching point of the decision maker with the midpoint of the probability interval. We can estimate specification (9) through a simple linear regression:

$$
p_{r}=\alpha p_{\text {mid }}+\beta+\varepsilon
$$

Given our design, we incorporate the between-subjects priming conditions in 
the following multiple regression:

$$
p_{r}=\left(\alpha_{n c}+\alpha_{o c} O C+\alpha_{u c} U C\right) p_{m i d}+\left(\beta_{n c}+\beta_{o c} O C+\beta_{u c} U C\right)+\varepsilon
$$

Where $\alpha$ is the ratio for the insensitivity indexes, and $\beta$ is the intercept for the neutral group. $O C, N C$, and $U C$ are dummy variables respectively for overconfident, neutral-confidence, and underconfident.

Estimates for each treatment group, significant at 5\% clustered on subjects. Estimates show that for the $\mathrm{NC}$ group, the ratio of slopes between ambiguity and risk weighting functions is $\alpha=.44(.22)$, while the term $\beta=.25(.10)$. For the OC group, the ratio of slopes is $\alpha=.98(.22), \beta=.019$ (.10) (similarly, we report the values for the UC group: $\alpha=.93(.22), \beta=.03$ (.10)). This result shows that for individuals in the neutral group, weighting functions for risk and ambiguity are further apart if compared to the overconfident group. For the OC decision makers, the weighting functions for risk and ambiguity tend to be closer to each other, having the ratio of slopes of these functions approaching the unit. These results confirm the magnitude and the direction of the ANOVA analysis.

\section{Discussion and Conclusions}

We believe that linking overconfidence and ambiguity attitudes is an important and timely issue, with immediate applications to the study of entrepreneurship, management, insurance markets, financial markets, and related fields.

Given the pervasiveness of uncertainty in business and entrepreneurial activities, we believe that addressing ambiguity attitudes of entrepreneurs is a timely issue. Recently, Pontikes (2012) claims that ambiguous situations are attractive to 
independent venture capitalists, since ambiguity allows entrepreneurs leeway for action aimed at meeting challenging and unclear targets. Conversely, institutional venture capitalists prefer clearly defined (non-ambiguous) targets and choose to fund mainstream projects. Even though the focus in Pontikes (2012) is not on probabilistic ambiguity, its contribution establishes the importance of addressing ambiguity in strategic management and entrepreneurship research. We could safely assume that ambiguity attitudes may constitute one antecedent factor of the effectuation logics theorized in Sarasvathy (2009). In her inspirational book, Sarasvathi suggests that entrepreneurs employ effectual constructivist logics in their decision making in situations where the future is unpredictable, goals are unclear, and the environment is driven by human action. This suggestions resonate with, and indirectly reinforce, the importance of ambiguity and ambiguity attitudes in entrepreneurial decision making.

Our results show that ambiguity attitudes are not a stable trait, and beyond domain and likelihood level, other factors may influence decision makers' attitude vis-à-vis ambiguity. Our conceptualization of overconfidence is broader than the "competence hypothesis" advanced by Heath and Tversky (1991). With our setting we confirm that overconfidence has a carry-over effect on ambiguity preferences in other domains. That is, individuals that experience a recent success due to their skill, would expand their positive belief to areas that are not directly related to skill. Yet, the performance of an investment project, may well depend on the abilities of the entrepreneurs, although not directly related to codified knowledge. We notice in particular that overconfident decision makers are consistent in their choices and persistently ambiguity seekers. Decision makers primed with neutral-confidence are ambiguity averse. Lastly, the underconfident group presents the most erratic behavior in terms of ambiguity preferences through experimental conditions. We find of extreme interest the reaction of overconfident 
decision makers to likelihood levels, degrees of ambiguity and stakes. Neutral and underconfident decision makers tend to show higher sensitivity in their ambiguity attitudes at changes in experimental conditions, i.e., magnitude of stakes.

It would be of great interest to relate business performance and entrepreneurial overconfidence, as well as innovativeness of the venture. It would be interesting to relate our results with observed excess entry (Camerer and Lovallo, 1999). If overconfident decision makers, on average correct likelihood insensitivity when deciding under ambiguity, then, is still overconfidence driving an irrational decision to enter a market? Rather, it could be the underconfident or neutral-confidence group, that behave as ambiguity seeking, that may account for high failure rates (as reported in Cooper et al. (1988)). Why underconfident decision makers would still exhibit ambiguity seeking behavior on investment decisions? This result could be driven by a sense of lack of control, escalation of commitment, loss aversion, or a feeling of "having nothing to lose".

Since March and Shapira (1987) contribution on managerial perspectives on risk attitudes, decision science and behavioral decision making have evolved in order to accommodate more complexity in the existing models, and incorporate as well the role of uncertainty in decision making. Thus, our research is informative to researchers in entrepreneurship and strategic decision making in several ways. It re-opens the debate on risk and risk attitudes, casting doubts that risk attitudes may not be capturing the entire observed variance on entrepreneurial behavior. Also, individuals and organizations are not rational decision makers, thus they process probabilistic information in ways that are susceptible to biases, psychological states, emotions, mood, etc.,. Moreover, this research is, hopefully, but a starting point of applications of decision theories to entrepreneurship and management studies. Strategic decisions are constantly made under conditions of ambiguity and calls for decision often do not allow to managers sufficient time to 
collect more useful information to support their choices. More often than not, information is not available. Are entrepreneurs individuals that are naturally prone to ambiguity? Or, individuals that learn to embrace uncertainty, adjust faster to the unexpected are the ones that become entrepreneurs?

Open questions in managerial and entrepreneurial cognition include inquiries related to whether individuals who tend to take risky or uncertain decisions in their professional lives also tend to underinsure. That is, is it the case that a person who can tolerate a high degree of uncertainty in the development of a new project would also refrain from insuring their property or their life, or will it be the case that people tend to be overconfident in their professional domain, yet to be uncertainty averse in their personal lives?

Other applications include managerial compensation schemes with variable components that depend on performance. Would overconfident managers seek more ambiguous compensation packages since they believe in higher future performance of their units? Or perhaps they feel more in control of certain events that determine pay?

Past research has documented that attitudes towards risk and ambiguity are not global features, but domain-specific. These questions suggest future directions of investigations aimed at assessing whether individual attitudes differ between situations in which the person has a sense of control (presumably, their choice of project) and situations in which the person does not (such as environmental contingencies).

These questions have had very little attention to date, especially in the field of entrepreneurship. This work brings in a novelty by jointly, yet independently, considering overconfidence and ambiguity attitudes in an experimental investigation with Executive Education participants that self-selected in the Entrepreneurship and Innovation track. To the best of our knowledge, this is the first work investi- 
gating entrepreneurial choice under ambiguity and the impact of overconfidence.

\section{Acknowledgments}

I would like to express my gratitude to professors Mohammed Abdellaoui, Thomas Astebro, Itzhak Gilboa, and Jamal Shamsie for their valuable comments. This work is partly funded by HEC Paris Leadership Center. Previous versions of this paper benefited from discussions with conference participants at EMLyon in Chamonix, ASFEE in Montpellier, FUR XV in Atlanta, ESA in New York, SEI in Copenhagen, and SMS in Prague.

\section{References}

Abdellaoui, M., Baillon, A., Placido, L., and Wakker, P. P. (2011). The rich domain of uncertainty: Source functions and their experimental implementation. The American Economic Review, 101(2):695-723.

Allais, M. (1953). Le comportement de l'homme rationnel devant le risque: Critique des postulats et axiomes de l'école américaine. Econometrica: Journal of the Econometric Society, pages 503-546.

Åstebro, T., Jeffrey, S. A., and Adomdza, G. K. (2007). Inventor perseverance after being told to quit: The role of cognitive biases. Journal of Behavioral Decision Making, 20(3):253-272.

Bernardo, A. E. and Welch, I. (2001). On the evolution of overconfidence and entrepreneurs. Journal of Economics $\&$ Management Strategy, 10(3):301-330.

Blavatskyy, P. R. (2009). Betting on own knowledge: Experimental test of overconfidence. Journal of Risk and Uncertainty, 38(1):39-49.

Bolger, F., Pulford, B. D., and Colman, A. M. (2008). Market entry decisions. Experimental Psychology (formerly Zeitschrift für Experimentelle Psychologie), $55(2): 113-120$.

Budescu, D. V., Kuhn, K. M., Kramer, K. M., and Johnson, T. R. (2002). Modeling certainty equivalents for imprecise gambles. Organizational Behavior and Human Decision Processes, 88(2):748-768.

Budner, S. (1962). Intolerance of ambiguity as a personality variable. Journal of personality. 
Burmeister, K. and Schade, C. (2007). Are entrepreneurs' decisions more biased? an experimental investigation of the susceptibility to status quo bias. Journal of Business Venturing, 22(3):340-362.

Camerer, C. and Lovallo, D. (1999). Overconfidence and excess entry: An experimental approach. The American Economic Review, 89(1):306-318.

Camerer, C. and Weber, M. (1992). Recent developments in modeling preferences: Uncertainty and ambiguity. Journal of risk and uncertainty, 5(4):325-370.

Carleton, R. N., Gosselin, P., and Asmundson, G. J. (2010). The intolerance of uncertainty index: Replication and extension with an english sample. Psychological assessment, 22(2):396-406.

Compte, O. and Postlewaite, A. (2004). Confidence-enhanced performance. American Economic Review, pages 1536-1557.

Cooper, A. C., Woo, C. Y., and Dunkelberg, W. C. (1988). Entrepreneurs' perceived chances for success. Journal of business venturing, 3(2):97-108.

Curley, S. P. and Yates, J. F. (1985). The center and range of the probability interval as factors affecting ambiguity preferences. Organizational behavior and human decision processes, 36(2):273-287.

De Bondt, W. F. and Thaler, R. H. (1994). Financial decision-making in markets and firms: A behavioral perspective. Technical report, National Bureau of Economic Research.

Dollinger, M. J. (1983). Use of budner's intolerance of ambiguity measure for entrepreneurial research. Psychological reports, 53(3):1019-1021.

Dollinger, M. J., Saxton, T., and Golden, P. A. (1995). Intolerance of ambiguity and the decision to form an alliance. Psychological reports, 77(3f):1197-1198.

Einhorn, H. J. and Hogarth, R. M. (1985). Ambiguity and uncertainty in probabilistic inference. Psychological review, 92(4):433.

Ellsberg, D. (1961). Risk, ambiguity, and the Savage axioms. The Quarterly Journal of Economics, pages 643-669.

Fischhoff, B., Slovic, P., and Lichtenstein, S. (1977). Knowing with certainty: The appropriateness of extreme confidence. Journal of Experimental Psychology: Human Perception and Performance, 3(4):552. 
Fox, C. R. and Tversky, A. (1995). Ambiguity aversion and comparative ignorance. The quarterly journal of economics, 110(3):585-603.

Freeston, M. H., Rhéaume, J., Letarte, H., Dugas, M. J., and Ladouceur, R. (1994). Why do people worry? Personality and Individual Differences, 17(6):791-802.

Frenkel-Brunswik, E. (1949). Intolerance of ambiguity as an emotional and perceptual personality variable. Journal of personality, 18(1):108-143.

Ghirardato, P., Maccheroni, F., and Marinacci, M. (2004). Differentiating ambiguity and ambiguity attitude. Journal of Economic Theory, 118(2):133-173.

Gigerenzer, G., Hoffrage, U., Kleinbölting, H., et al. (1991). Probabilistic mental models: A brunswikian theory of confidence. Psychological review, 98(4):506528.

Gilboa, I. (2009). Theory of Decision under Uncertainty. Cambridge University Press.

Gilboa, I. and Schmeidler, D. (1989). Maxmin expected utility with non-unique prior. Journal of mathematical economics, 18(2):141-153.

Grenier, S., Barrette, A.-M., and Ladouceur, R. (2005). Intolerance of uncertainty and intolerance of ambiguity: Similarities and differences. Personality and individual differences, 39(3):593-600.

Grieco, D. and Hogarth, R. M. (2009). Overconfidence in absolute and relative performance: The regression hypothesis and bayesian updating. Journal of Economic Psychology, 30(5):756-771.

Griffin, D. and Tversky, A. (1992). The weighing of evidence and the determinants of confidence. Cognitive psychology, 24(3):411-435.

Hamilton, B. H. (2000). Does entrepreneurship pay? an empirical analysis of the returns to self-employment. Journal of Political Economy, 108(3):604-631.

Hayward, M. L., Shepherd, D. A., and Griffin, D. (2006). A hubris theory of entrepreneurship. Management Science, 52(2):160-172.

Headd, B. (2003). Redefining business success: Distinguishing between closure and failure. Small Business Economics, 21(1):51-61.

Heath, C. and Tversky, A. (1991). Preference and belief: Ambiguity and competence in choice under uncertainty. Journal of risk and uncertainty, 4(1):5-28. 
Heller, Y. (2011). Overconfidence and diversification. Available at SSRN 1691790.

Hilary, G. and Menzly, L. (2006). Does past success lead analysts to become overconfident? Management Science, 52(4):489-500.

Hoelzl, E. and Rustichini, A. (2005). Overconfident: Do you put your money on it?*. The Economic Journal, 115(503):305-318.

Kahneman, D. and Tversky, A. (1979). Prospect theory: An analysis of decision under risk. Econometrica: Journal of the Econometric Society, pages 263-291.

Keynes, J. (1921). oa treatise on probabilityv. London: Macmillan\&Co.

Kilka, M. and Weber, M. (2001). What determines the shape of the probability weighting function under uncertainty? Management science, 47(12):1712-1726.

Klibanoff, P., Marinacci, M., and Mukerji, S. (2005). A smooth model of decision making under ambiguity. Econometrica, 73(6):1849-1892.

Knight, F. (1921). Risk, Uncertainty, and Profit. New York: Kelley and Millman.

Koellinger, P., Minniti, M., and Schade, C. (2007). I think I can, I think I can: Overconfidence and entrepreneurial behavior. Journal of Economic Psychology, 28(4):502-527.

Krueger, N. and Dickson, P. R. (1994). How believing in ourselves increases risk taking: Perceived self-efficacy and opportunity recognition. Decision Sciences, 25(3):385-400.

Mac Donald Jr, A. (1970). Revised scale for ambiguity tolerance: Reliability and validity. Psychological reports, 26(3):791-798.

March, J. G. and Shapira, Z. (1987). Managerial perspectives on risk and risk taking. Management science, 33(11):1404-1418.

McLAIN, D. L. (2009). Evidence of the properties of an ambiguity tolerance measure: The multiple stimulus types ambiguity tolerance scale-ii (mstat-ii) 1 . Psychological reports, 105(3):975-988.

Moore, D. A. and Healy, P. J. (2008). The trouble with overconfidence. Psychological review, 115(2):502.

Pontikes, E. G. (2012). Two sides of the same coin how ambiguous classification affects multiple audiences evaluations. Administrative Science Quarterly, 57(1):81-118. 
Pulford, B. D. (1996). Overconfidence in human judgement. University of Leicester.

Sarasvathy, S. D. (2009). Effectuation: Elements of entrepreneurial expertise. Edward Elgar Publishing.

Schade, C. and Burmeister, K. (2009). Experiments on Entrepreneurial Decision Making, volume 5. Now Publishers Inc.

Schere, J. L. (1982). Tolerance of ambiguity as a discriminating variable between entrepreneurs and managers. In Academy of management proceedings, volume 1982, pages 404-408. Academy of Management.

Schmeidler, D. (1989). Subjective probability and expected utility without additivity. Econometrica: Journal of the Econometric Society, pages 571-587.

Stankov, L. (2000). Complexity, metacognition, and fluid intelligence. Intelligence, 28(2):121-143.

Tajeddini, K. and Mueller, S. L. (2009). Entrepreneurial characteristics in switzerland and the uk: a comparative study of techno-entrepreneurs. Journal of International Entrepreneurship, 7(1):1-25.

Teoh, H. Y. and Foo, S. L. (1997). Moderating effects of tolerance for ambiguity and risktaking propensity on the role conflict-perceived performance relationship: evidence from singaporean entrepreneurs. Journal of business Venturing, 12(1):67-81.

Van den Steen, E. (2011). Overconfidence by bayesian-rational agents. Management Science, 57(5):884-896.

Wakker, P. P. (2004). On the composition of risk preference and belief. Psychological Review, 111(1):236-241.

Wakker, P. P. (2010). Prospect theory: For risk and ambiguity. Cambridge University Press.

Wu, G. and Gonzalez, R. (1996). Curvature of the probability weighting function. Management science, 42(12):1676-1690.

Wu, G. and Gonzalez, R. (1999). Nonlinear decision weights in choice under uncertainty. Management Science, 45(1):74-85. 